\title{
Algorithm of Image Definition Evaluation Based on Lifting Scheme
}

\author{
Jing Zhang \\ Key Laboratory of Optical Engineering, Chinese Academy \\ of Sciences, Chengdu, 610200, China \\ Graduate University of Chinese Academy of Sciences, \\ Beijing, 100000, China
}

\author{
Ke Chen, Sheng-ping Du, Yi-han Luo \\ Key Laboratory of Optical Engineering, Chinese Academy \\ of Sciences, Chengdu, 610200, China
}

\begin{abstract}
Due to the existing image definition evaluation functions in auto-focus systems easily affected by noise, an image definition evaluation algorithm based on lifting scheme is proposed. The algorithm uses lifting scheme for wavelet decomposition, and adopts the energy value of high frequency components of wavelet decomposition as image definition evaluation function. Theoretical analysis and extensive simulation results show that this method, compared with other image definition evaluation functions, has higher sensitivity, excellent anti-noise performance, low computation burden and high reliability, so it can better meet the requirements of image definition evaluation.
\end{abstract} focus.

Keywords-image definition evaluation, lifting scheme, auto-

\section{INTRODUCTION}

Optical imaging systems as a tool of acquiring image information have been widely used in industry, agriculture, medicine, military and other fields. Whether a camera in everyday life or photoelectric measurement equipment in military field, optical imaging systems play an extremely important role[1]. Any optical imaging system can meet the focusing question, and then determining the clarity of digital images is also paid more and more widespread attention. Digital image evaluation function is an important basis for the evaluation of digital image clarity, which its performance directly determines the accuracy and speed of auto-focus.

Ideal evaluation function should have following characteristics[2,3]: unbiasedness, namely, the extreme position corresponding the clearly focusing point of the image; monotonicity, that is to say the curve has one and only one global extremum; high sensitivity, that means the algorithm can distinguish the image with little defocus; immunity, namely, adapting to the complex and changing environment; small computational complexity etc.. Domestic and foreign scholars for auto-focus system of different applications have done a lot of research, and produced a lot of image definition evaluation functions, mainly including the following four categories[4]: (1) spatial domain evaluation function, such as energy gradient operator function, Roberts operator function, Tenengrad function etc.; (2) frequency domain evaluation function, such as Fourier transform, Discrete Cosine transform, wavelet transform etc.; (3) informatics evaluation function, such as entropy function; (4) statistics evaluation function, such as Range function, autocorrelation function. The study found that the spatial domain evaluation function has simple calculation, low complexity and good real-time performance; frequency domain evaluation function has high sensitivity, intensive computation, and is difficult to achieve real-time performance; informatics and statistics evaluation function are rarely used, for which are sensitive on the environment, lighting etc.. The worst thing is that once these algorithms suffer the effects of noise, there will be large fluctuations, and sensitivity and robustness is reduced, which have a serious impact on the accuracy and reliability of the evaluation results. To solve these problems, this paper proposes a definition evaluation function based on lifting scheme. Compared with other functions, this method has a better anti-noise performance and sensitivity, low computational complexity, and easily implements realtime performance.

\section{IMAGE DEFINITION EVALUATION BASED ON LIFTING SCHEME}

\section{A The principle of lifting wavelet transform}

Assuming that the size of two-dimensional digital image is $M^{*} \mathrm{~N}$, the image's discrete wavelet transform is expressed as follows[5]:

$$
\begin{aligned}
& W_{\phi}(j, m, n)=\frac{1}{\sqrt{M N}} \sum_{x=0}^{M-1} \sum_{y=0}^{N-1} f(x, y) \phi_{j, m, n}(x, y) \\
& W_{\psi}^{H, V, D}(j, m, n)=\frac{1}{\sqrt{M N}} \sum_{x=0}^{M-1} \sum_{y=0}^{N-1} f(x, y) \psi_{j, m, n}^{H, V, D}(x, y)
\end{aligned}
$$

Where, $\varphi_{j, m, n}, \psi_{j, m, n}$ are wavelet functions of $\mathrm{j}$ scales, the former is used to wavelet decomposition of maximum resolution, the latter is used to decomposition of horizontal direction, vertical direction and diagonal direction; $W_{\varphi}(j, m, n)$ is low frequency information coefficient of $\mathrm{j}$ scales; $W_{\psi}^{H, V, D}(j, m, n)$ are high frequency information coefficients of horizontal direction, vertical direction and diagonal direction of $\mathrm{j}$ scales, $H, V, D$ respectively represent horizontal direction, vertical direction and diagonal direction.

Wavelet transform is a local transformation of time and frequency. It has the advantage of multiresolution analysis, thereby focusing to any details of the signal, which makes it possible to distinguish the image with little defocus, so 
greatly improves the accuracy of the auto-focus[6,7]. But when the noise is relatively large, evaluation result is easy to make an error. And generally the wavelet transform is a convolution operation, which leads to intensive computation, high computational complexity and storage space requirements, and is not conducive to realize real-time performance. So it is necessary to improve the function of traditional wavelet transform so that establish a definition evaluation function of fast speed and effectively suppressing noise.

Sweldens, in 1994, proposed a new wavelet construction program - lifting scheme, becoming the basic tool of constructing the second generation wavelet (also called lifting wavelet)[8,9,10,11]. A significant advantage of lifting scheme is to improve the speed of wavelet transform. According to Daubechies' analysis, with the length of filter increasing, the computational speed tends to be two times as traditional wavelet transform. This method of constructing wavelet doesn't need the condition of translation and scaling of binary wavelet function, does not depend on the Fourier transform, and converts the signal completely at spatial domain, but has all advantages of traditional wavelet transform. The whole operation process does not need to apply for secondary storage space, so we can save storage resources.

The entire lifting scheme separates high frequency and low frequency of signals by three steps: lazy wavelet transform, dual lifting, lifting.

- Lazy wavelet transform

The original signal $S_{j}$ is split into two subsets, and the general split method is parity split. The even set $S_{j, 2 l}$ contains all even values of the original signal, and the odd set $S_{j, 2 l+1}$ contains all odd values of the original signal.

$$
S_{j}=(\text { even, odd })=\left(S_{j, 2 l}, S_{j, 2 l+1}\right)
$$

- $\quad$ Dual lifting

Dual lifting process is based on the correlation between the data. Because an odd value and an even value have a certain correlation, we can use the value of the even part to predict the value of the odd part.

$$
d_{j-1}=S_{j, 2 l+1}-P\left(S_{j, 2 l}\right)
$$

Where, $d_{j-1}$ is the residual signal, corresponding to the high frequency part of wavelet transform; $\mathrm{P}$ is the dual lifting operator.

- $\quad$ Lifting

Some properties of the subset $S_{j, 2 l}$ are different from the original data, so it is necessary to lifting, finally generating a subset $C_{j-1}$, which is consistent with the original signal:

$$
c_{j-1}=S_{j, 2 l}-U\left(d_{j-1}\right)
$$

Where, $c_{j-1}$ corresponds to the low frequency part of wavelet transform. $\mathrm{U}$ is the lifting operator.
Repeating the above steps, we can obtain multilevel wavelet transform of the original signal.

\section{B Image definition evaluation function based on lifting wavelet transform}

D5/3 biorthogonal wavelet is widely used in the image processing. As an example, its corresponding algorithm is expressed as follows[12]:

$$
\begin{aligned}
& s_{j}^{(0)}=x_{2 l} \\
& d_{j}^{(0)}=x_{2 l+1} \\
& d_{j}^{(1)}=d_{j}^{(0)}+\left\lfloor\tau\left(s_{j}^{(0)}+s_{j+1}^{(0)}\right)+1 / 2\right\rfloor \\
& s_{j}^{(1)}=s_{j}^{(0)}+\left\lfloor v\left(d_{j}^{(1)}+d_{j+1}^{(1)}\right)+1 / 2\right\rfloor
\end{aligned}
$$

Where, $x$ is the original signal, $s$ is the low frequency information, $d$ is high frequency information, $\tau, v$ are the dual lifting operator and lifting operator, $\tau=-0.5, v=0.25$.

When carrying out lifting wavelet transform for an image, firstly line conversion is conducted and the image is decomposed into a low frequency band and a high frequency band; then the two bands were converted separately by columns, and the image is decomposed into four bands: a low frequency band $L L_{1}$ (upper left corner) and three high-frequency band: $H L_{1}$ (upper right corner), $\mathrm{LH}_{1}$ (lower left corner), $H_{H_{1}}$ (lower right corner).

\begin{tabular}{|c|c|c|}
\hline$L L_{2}$ & $H L_{2}$ & \\
\hline $\mathrm{LH}_{2}$ & $\mathrm{HH}_{2}$ & $H L_{1}$ \\
\hline \multicolumn{2}{|c|}{$L H_{1}$} & $H H_{1}$ \\
\hline
\end{tabular}
$H L_{1}, L H_{1}, H H_{1}$ respectively represent the three high frequency components of horizontal direction, vertical direction and diagonal direction of the first layer wavelet decomposition. Repeat these steps for the low frequency band to complete the second layer lifting wavelet decomposition as shown in Fig.1.

Figure 1. The second layer wavelet decomposition

Because the focusing process is the energy variation process of the image, an precise focusing image contains the largest energy, and the high frequency coefficients of the second layer wavelet composition can better characterize image clarity and have better anti-noise performance. So take the energy of high frequency components of the second layer wavelet composition as a definition evaluation operator, and its formula is expressed as follows: 


$$
f=\sum\left(W_{\mathrm{HL}_{2}}^{2}+W_{\mathrm{LH}_{2}}^{2}+W_{\mathrm{HH}_{2}}^{2}\right)
$$

Where, $W_{\mathrm{HL}_{2}}, W_{\mathrm{LH}_{2}}, W_{\mathrm{HH}_{2}}$ are high frequency information coefficients of horizontal direction, vertical direction and diagonal direction of the second layer wavelet decomposition.

\section{EXPERIMENTAL RESULTS AND ANALYSIS}

In order to observe the performance of various types of evaluation functions, the thirteen different defocus states' lena images are evaluated using MATLAB on the PC. Where, the seventh image is the focusing image. Having better performance's energy gradient function, Brenner function, traditional wavelet transform are selected to make a comparison with lifting wavelet transform in conditions of the absence of noise and noise (Gaussian white noise with zero mean and variance of 0.005). Simulation curves of evaluation functions are shown in Fig.2 and Fig.3.

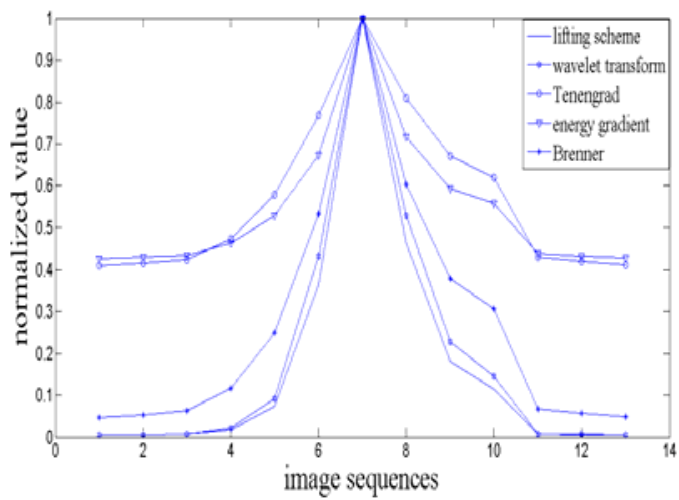

Figure 2. Evaluation curves in noiseless condition

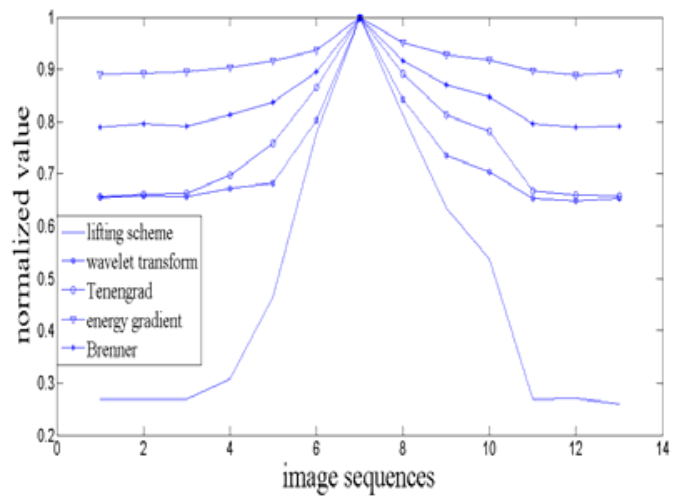

Figure 3. Evaluation curves in noisy condition

The formula of the sensitivity is expressed as follows:

$$
S=\sum_{i=1}^{12}|F(i+1)-F(i)|
$$

Where, $F(i)$ is the definition evaluation value of image $i ; i$ is image's number.

According to the above formula, we can calculate the sensitivity values of various image definition evaluation functions. The result is shown in Table 1.

Table I. THE SENSITIVITY VALUES OF VARIOUS EVALUATION FUNCTIONS

\begin{tabular}{|c|c|c|c|c|c|}
\hline $\begin{array}{c}\text { Evaluation } \\
\text { functions }\end{array}$ & $\begin{array}{c}\text { Lifting } \\
\text { wavele } \\
\mathrm{t}\end{array}$ & $\begin{array}{c}\text { Tradition } \\
\mathrm{al} \\
\text { wavelet }\end{array}$ & $\begin{array}{c}\text { Tenengra } \\
\mathrm{d}\end{array}$ & $\begin{array}{c}\text { Energy } \\
\text { gradient }\end{array}$ & $\begin{array}{c}\text { Brenne } \\
\mathrm{r}\end{array}$ \\
\hline $\begin{array}{c}\text { Sensitivity } \\
\text { (noiseless } \\
\text { ) }\end{array}$ & 1.9906 & 1.9898 & 1.1797 & 1.1482 & 1.9054 \\
\hline $\begin{array}{c}\text { Sensitivity } \\
\text { (noisy) }\end{array}$ & 1.4929 & 0.7366 & 0.6852 & 0.2297 & 0.4227 \\
\hline
\end{tabular}

Fig. 2 and Table 1 show that the curve of the evaluation function based on lifting wavelet has maximum slope on both sides of the peak and the best acuteness, so sensitivity is the highest; when adding Gaussian noise, due to the random of noise, it is the equivalent with adding a random amount. Fig.3 and Table 1 show all of the evaluation function curves have a "floating" phenomenon, a sharp deterioration, so that sensitivity is reduced. However, after adding the images with noise, compared to other evaluation functions, evaluation function curve based on lifting wavelet transform rises in the minimum, and still maintains good sharpness, namely, relatively high sensitivity. The sensitivity's change is small, that is to say lifting wavelet has good anti-noise performance. Therefore, lifting wavelet transform has higher sensitivity and better anti-noise performance.

\section{CONCLUSIONS}

In the case that image definition evaluation function is very important in auto-focus systems, this paper proposes an image definition evaluation function based on lifting wavelet. Theoretical analysis and extensive simulation experiments show that the evaluation function proposed in this paper has the highest sensitivity and robustness, and is fully carried out at spatial domain. Compared to the traditional wavelet transform, computational speed is doubled and complexity is greatly reduced. The image definition evaluation function based on lifting scheme is an accurate and stable algorithm, which can provide a reliable guarantee for the subsequent processing of the image.

\section{REFERENCES}

[1] Huang De-Tian, Study on Auto-focusing Method Using Image Technology, Changchun Institute of Optics, Fine Mechanics and Physics Chinese Academy of Sciences, 2013.

[2] Lin Zhao-Hua, Study on the application of the autofocus technology based on the imagine processing in the theodolite, Changchun Institute of Optics, Fine Mechanics and Physics Chinese Academy of Sciences, 2012, 1-128.

[3] Lu Zhen-Hua, The Automatic Image-based Real-time Focusing Research in Pushbroom Remote Sensing Camera, Changchun Institute of Optics, Fine Mechanics and Physics Chinese Academy of Sciences, 2012, 1-146. 
[4] Wang Jian, Study on Technology of Auto-focus Based on Image Processing, Institute of Optics and Electronics, Chinese Academy of Sciences, 2013.

[5] Ma Shu-Xiao, Studies on Auto-focus System based on Lifting DWT, Shandong University, 2013.

[6] Gilber Strang, Truong Nguyen. Wavelets and Filters Banks. WellesleyCambridge Press. Box 812060. Wellesley MA 02181, 1-515.

[7] Wang Xin, Liu Chang, Algorithm of image definition evaluation based on lifting wavelet transform, Journal of Northeast Normal University(Natural Science Edition), 2009,41(4):52-59.

[8] DAUBECHIES I, SWELDENS W. Factoring wavelet transforms into lifting steps[J]. J Fourier Anal, 1998, 4(3):245-267.
[9] SWELDENS. The lifting scheme: a construction of second generation wavelets[J]. SIA M J Math Anal, 1997, 29(2):511-546.

[10]Daubechies I. Ten Lectures on Wavelets. CBMS-NSF Regional Conf. Series in Appl. Math,Vol. 61. Society for Industrial and Applied Mathematics, Philadelphia, 1992: 215-249.

[11]Wu Yong-Hong, Pan Quan, Wavelet Transform that Map Integers to Integers Based on Lifting Scheme. Journal of Electronics \& Information Technology, 2004, 26(4):659-663.

[12]Li F, Jin H. A Fast Auto-focusing method for digital still camera. Proceedings of the Fourth International Conference on Machine Learning and Cybernetics [C]. Guangzhou, IEEE, 2005: P5001-5005. 